\title{
WEISS Criteria
}

National Cancer Institute

\section{Source}

National Cancer Institute. WEISS Criteria. NCI Thesaurus. Code C104015.

Histopathologic criteria to evaluate adrenocortical tumors. These criteria include nuclear grade III or IV, mitotic rate greater than five per fifty high power fields, presence of atypical mitotic figures, presence of less than or equal to twenty five percent of clear or vacuolated cells that resemble normal zona fasciculata, diffuse architecture, necrosis, venous invasion, sinusoid invasion, and invasion of tumor capsule. 